\title{
Tsallis power laws and finite baths with negative heat capacity
}

\author{
G. Baris Bagci \\ Department of Physics, Faculty of Science, Ege University, 35100 Izmir, Turkey \\ Thomas Oikonomou* \\ Department of Physics, University of Crete, 71003 Heraklion, Greece \\ and Department of Physics, Faculty of Science, Ege University, 35100 Izmir, Turkey
}

(Received 11 May 2013; published 14 October 2013)

\begin{abstract}
It is often stated that heat baths with finite degrees of freedom i.e., finite baths, are sources of Tsallis distributions for classical Hamiltonian systems. By using well-known fundamental statistical mechanics expressions, we rigorously show that Tsallis distributions with fat tails are possible only for finite baths with constant negative heat capacity, while constant positive heat capacity finite baths yield decays with sharp cutoff with no fat tails. However, the correspondence between Tsallis distributions and finite baths holds at the expense of violating the equipartition theorem for finite classical systems at equilibrium. We comment on the implications of the finite bath for the recent attempts towards a $q$-generalized central limit theorem.
\end{abstract}

DOI: 10.1103/PhysRevE.88.042126

PACS number(s): 05.70.Ce, 05.70.Ln

\section{INTRODUCTION}

Recently, a great deal of effort has been put into the construction of a nonextensive thermostatistics based on the Tsallis entropies

$$
S_{q}(p)=\frac{\int d \Gamma p^{q}(\Gamma)-1}{1-q},
$$

where $\Gamma$ and $p(\Gamma)$ denote the phase space variables and the probability distribution, respectively [1]. The Tsallis $q$ distributions are obtained from the maximization of the Tsallis entropies by the internal energy $E$ calculated from either $E=\int d \Gamma p(\Gamma) H$ or

$$
E=\frac{\int d \Gamma p^{q}(\Gamma) H}{\int d \Gamma p^{q}(\Gamma)}
$$

where $H$ is the Hamiltonian. The former are called ordinary Tsallis distributions and are of the form $1 / \exp _{q}\left(\gamma_{q} H\right)\left(\gamma_{q}\right.$ being a positive constant) apart from normalization, where $\exp _{q}(x)=[1+(1-q) x]_{+}^{1 /(1-q)}$ with $[x]_{+}=\max \{0, x\}$. The latter are escort Tsallis distributions of the form $\exp _{q}\left(-\gamma_{q} H\right)$, omitting the normalization constant.

Despite these efforts, however, the true origin of Tsallis distributions in statistical mechanics is elusive. In contrast, there seems to be agreement on an important and very intuitive statistical mechanics source of Tsallis distributions, namely, heat baths with a finite number of degrees of freedom, simply called finite baths [1-9]. A brief history of the previous attempts is important to shed light on this extensively researched subject and to determine the context of the present paper among them. An early work that drew attention to the uses of finite baths for Tsallis statistics is Ref. [2]. The importance of the finite baths in this context for negative heat capacities was mentioned in Refs. [3,4], while emphasis of the requirement of ergodicity for finite baths in particular is found in Ref. [5]. Their use to interpolate between the microcanonical and canonical ensembles without a violation of the equipartition

*Corresponding author: thoik@ physics.uoc.gr theorem was further elaborated in Refs. [6,7]. However, despite the distribution being $q$ exponential, Ref. [7] notes that the associated entropy is the Rényi entropy, which is additive, contrary to the Tsallis entropy. In this connection, Ref. [8] is noteworthy in showing that the entropy can be either Tsallis or Rényi entropy depending on whether the thermodynamic probability of the isolated system or the canonical (finite) subsystem-reservoir probability is factorized. The possible role of Rényi entropy for finite baths is further, albeit implicitly, suggested in [9], since a superstatistical approach shows that the relevant entropy for the finite baths seems to be additive.

According to this view, a system coupled to a finite heat bath attains an inverse power-law distribution in the form of $q$-exponential decays for any of the two branches of the nonextensivity parameter $q: q>1$ and $q<1$. The heat capacity of the bath can be found as $\frac{1}{1-q}$ or $\frac{1}{q-1}$, depending on which Tsallis distributions are used, i.e., ordinary or escort. Since the range of admissible $q$ values can be both above and below the value $q=1$, the heat bath can have both positive and negative heat capacity values. Despite seeming counterintuitive at first, the possibility of systems with negative heat capacity was first pointed out by Lynden-Bell and Wood $[10,11]$ and later investigated theoretically by Thirring, who showed that microcanonical ensembles can in fact have negative specific heats [12]. Important contributions in this context have also been presented by Gross, who emphasized the fact that the microcanonical ensembles are fundamental since they do not rely on the assumptions of additivity or extensivity [13]. Recently, negative heat capacity expressions have also been found in one-dimensional evaporation models and long-range quantum spin systems in optical lattices treated as microcanonical ensembles [14,15]. Experimental measurements of the negative microcanonical heat capacity have been carried out with small clusters of sodium atoms near the solid to liquid transition and liquid to gas transition for the cluster of hydrogen ions $[16,17]$. As expected, when $q=1, q$-exponential decays become ordinary exponential distributions and simultaneously the heat bath attains infinite heat capacity $[3,4]$. 
In order to obtain the aforementioned results, one assumes that a subsystem (simply called system from now on) embedded in a finite heat bath interacts weakly and together they form the total system. Then the total system can be treated microcanonically. In treating subsystems in contact with an infinite reservoir, one can choose either the phase space surface $\Omega$ or volume $\Phi$ as the appropriate measure without loss of generality since these two yield the same results in the thermodynamic limit [18]. However, when dealing with systems of finite degrees of freedom, one should consider two phase space measures separately since these two measures might yield different results. Therefore, a rigorous study of the finite baths must take into account both ordinary and escort Tsallis distributions and phase space volume and surface demarcation into account.

The paper is organized as follows. In the next section we outline the general microcanonical approach that will be used throughout the paper. In Sec. III we present the results by considering all possibilities, i.e., ordinary and escort distributions as well as phase space volume and surface demarcations together with the positivity or negativity of the total energy. Finally, a summary is presented in Sec. IV.

\section{MICROCANONICAL APPROACH}

We begin by considering a system weakly coupled to another one acting as a (finite) bath so that the total Hamiltonian is assumed to be ergodic. Despite the presence of the interaction between the system and bath, the total system, i.e., system plus bath, is assumed to be isolated. The Hamiltonian of the total system is given as

$$
H_{\mathrm{tot}}(\mathbf{x}, \mathbf{p}, \mathbf{X}, \mathbf{P})=H_{S}(\mathbf{x}, \mathbf{p})+H_{B}(\mathbf{X}, \mathbf{P})+h(\mathbf{x}, \mathbf{X}),
$$

where $H_{S}(\mathbf{x}, \mathbf{p})$ and $H_{B}(\mathbf{X}, \mathbf{P})$ denote the system and bath Hamiltonians, respectively, interacting with one another through the interaction term $h(\mathbf{x}, \mathbf{X})$. The system and bath phase space coordinates are respectively given by $\mathbf{x}=$ $\left\{\mathbf{x}_{1}, \ldots, \mathbf{x}_{N_{S}}\right\}, \mathbf{p}=\left\{\mathbf{p}_{1}, \ldots, \mathbf{p}_{N_{S}}\right\}$ and $\mathbf{X}=\left\{\mathbf{X}_{1}, \ldots, \mathbf{X}_{N_{B}}\right\}, \mathbf{P}=$ $\left\{\mathbf{P}_{1}, \ldots, \mathbf{P}_{N_{B}}\right\}$, where $\left\{\mathbf{x}_{i}, \mathbf{p}_{i}\right\}_{i=1}^{N_{S}},\left\{\mathbf{X}_{i}, \mathbf{P}_{i}\right\}_{i=1}^{N_{B}} \in \mathbb{R}^{D}, D$ being the dimensionality of the space. The system and bath Hamiltonians in particular read

$$
\begin{gathered}
H_{S}(\mathbf{x}, \mathbf{p})=\sum_{i=1}^{N_{S}} \frac{\mathbf{p}_{i}^{2}}{2 m}+\mathcal{V}_{S}(\mathbf{x}), \\
H_{B}(\mathbf{X}, \mathbf{P})=\sum_{i=1}^{N_{B}} \frac{\mathbf{P}_{i}^{2}}{2 M}+\mathcal{V}_{B}(\mathbf{X}),
\end{gathered}
$$

where $\mathcal{V}_{S}(\mathbf{x})$ and $\mathcal{V}_{B}(\mathbf{X})$ are the interactions within the system and bath. From here on, the positivity of the system energy $E_{S}$ is assumed. Moreover, the Boltzmann constant is set equal to dimensionless unity so that the temperature has the same dimension as energy.

The marginal probability distribution $p(\mathbf{x}, \mathbf{p})$ of finding the system $S$ in a particular state with positive energy $E_{S}$ reads [18]

$$
\begin{aligned}
p(\mathbf{x}, \mathbf{p}) & =\frac{\Omega_{B}\left(E_{\mathrm{tot}}-H_{S}(\mathbf{x}, \mathbf{p})\right)}{\Omega_{\mathrm{tot}}\left(E_{\mathrm{tot}}\right)} \\
& =c \Omega_{B}\left(E_{\mathrm{tot}}-H_{S}(\mathbf{x}, \mathbf{p})\right),
\end{aligned}
$$

where $c$ is the normalization constant given by the inverse of the density of states of the total system, i.e.,

$$
\Omega_{\mathrm{tot}}\left(E_{\mathrm{tot}}\right)=\int d \mathbf{x} d \mathbf{p} d \mathbf{X} d \mathbf{P} \delta\left(E_{\mathrm{tot}}-H_{\mathrm{tot}}(\mathbf{x}, \mathbf{p}, \mathbf{X}, \mathbf{P})\right) .
$$

Here $\delta$ denotes the Dirac delta function. Similarly, the density of states of the bath is given by

$$
\Omega_{B}\left(E_{B}\right)=\int d \mathbf{X} d \mathbf{P} \delta\left(E_{B}-H_{B}(\mathbf{X}, \mathbf{P})\right),
$$

where $E_{B}$ is the energy of the finite bath. Before proceeding, we further assume that the finite bath has constant heat capacity, i.e., $C_{B}=\frac{E_{B}}{T_{B}}$. However, the heat capacity of the system, in full generality, is given by the expression $C_{S}\left(T_{S}\right)=$ $\frac{\partial E_{S}\left(T_{S}\right)}{\partial T_{S}}, T_{S}$ being the temperature of the system. The system temperature becomes equal to that of the bath only when the heat capacity of the bath is infinite.

The probability distribution $p(\mathbf{x}, \mathbf{p})$ of the system in Eq. (3), due to the expression of the density of states of the bath in Eq. (5), involves the Dirac $\delta$ function, which is even, i.e., $\delta(x)=\delta(-x)$. Therefore, the Dirac $\delta$ function enforces two distinct cases: either $E_{\text {tot }}-H_{S} \geqslant 0$ or $E_{\text {tot }}-H_{S}<0$. Since the marginal system distribution is finally obtained by identifying $E_{B}=E_{\text {tot }}-H_{S}$, one must consider both cases of the finite bath possessing constant positive and negative energies, or due to the relation $C_{B}=\frac{E_{B}}{T_{B}}$, constant positive or negative finite heat capacities. It is important to understand the constraints imposed on the system's probability distribution due to these two possibilities: Considering the case $E_{\text {tot }}-H_{S} \geqslant 0$, one can see that the system energy at most can be equal to $E_{\text {tot }}$. This in turn implies that the probability distribution of the system in weak contact with a finite bath possessing positive heat capacity must have a cutoff at $H_{S}=E_{\text {tot }}$. This constraint excludes the possibility of the system distribution having fat tails. Therefore, if fat power-law tails should ever emerge in this context, this must be the case when the constant heat capacity of the finite bath (or equivalently the heat bath energy $\left.E_{B}\right)$ is negative, i.e., $E_{\text {tot }}-H_{S}<0$.

In order to proceed further, one must have an explicit expression for the density of states of the bath composed of finite classical particles. This expression can be shown to have the form $\Omega_{B}\left(E_{B}\right) \sim\left|E_{B}\right|^{k}$ with exponent $k$ apart from some multiplicative positive constant $[3,4,19]$. The absolute value is needed to ensure the positivity of the density of states. Calculating the temperature of the finite bath with $T_{B}^{-1}=\frac{\partial \ln \left(\Omega_{B}\right)}{\partial E_{B}}$ and comparing it with the expression $C_{B}=\frac{E_{B}}{T_{B}}$, we see that the exponent $k$ is equal to the finite heat capacity of the bath $C_{B}$ so that

$$
\Omega_{B}\left(E_{B}\right) \sim\left|E_{B}\right|^{C_{B}}
$$

where the finite heat bath capacity can be positive (i.e., $E_{\text {tot }}-$ $H_{S} \geqslant 0$ ) or negative (i.e., $E_{\text {tot }}-H_{S}<0$ ).

At this point, we remind the reader of an important fact: One should consider both the density of states $\Omega$ and the volume of the phase space $\Phi$ since these two measures might yield different results when dealing with systems of finite degrees of freedom although they are equivalent in the thermodynamic limit [20]. The two measures are related to one another by

$$
\Omega(E)=\frac{\partial \Phi(E)}{\partial E} .
$$


The equation above yields $\Phi_{B}\left(E_{B}\right) \sim\left|E_{B}\right|^{C_{B}+1}$ for a constant heat capacity bath. Therefore, by using $T_{B}^{-1}=\frac{\partial \ln \left(\Phi_{B}\right)}{\partial E_{B}}$ and comparing with the expression $C_{B}=\frac{E_{B}}{T_{B}}$, we obtain an important relation

$$
C_{B}^{\Omega}+1=C_{B}^{\Phi},
$$

which is valid as long as the heat capacity of the finite bath is constant. The superscripts denote the quantities calculated through the density of states $\Omega \equiv \Omega_{B}$ or phase space volume $\Phi \equiv \Phi_{B}$.

\section{FINITE BATH AND TSALLIS DISTRIBUTIONS}

Having outlined the general microcanonical approach to the totality of the system plus bath, we now explore the constant positive and negative heat capacity possibilities distinctly.

\section{A. Case $\boldsymbol{E}_{\boldsymbol{B}} \geqslant 0$}

This case, i.e., $E_{\mathrm{tot}}-H_{S} \geqslant 0$, corresponds to the marginal system distribution stemming from the identification $E_{B}=$ $E_{\text {tot }}-H_{S} \geqslant 0$. In other words, the (finite) system is now coupled to a finite bath with positive energy and therefore constant positive heat capacity $C_{B}$. Therefore, the system energy can attain at most the value $E_{\text {tot }}$, for which a necessary cutoff condition has to be respected in the probability distribution of the system. In fact, using Eqs. (3) and (6), we obtain the marginal probability distribution of the system

$$
p(\mathbf{x}, \mathbf{p})=c\left(E_{\mathrm{tot}}-H_{S}(\mathbf{x}, \mathbf{p})\right)^{C_{B}^{\Omega}},
$$

This distribution can be cast into the form of an escort $q$ exponential by identifying $\alpha_{q}^{-1}:=(1-q) E_{\mathrm{tot}}$ and

$$
C_{B}^{\Omega}=\frac{1}{1-q}
$$

so that

$$
p(\mathbf{x}, \mathbf{p}) \sim \exp _{q}\left(-\alpha_{q} H_{S}(\mathbf{x}, \mathbf{p})\right)
$$

apart from the normalization. Considering consistently the conditions above together with $\alpha_{q}>0$, we obtain the following range of validity for the nonextensivity index $q$ :

$$
\left.\left.\begin{array}{l}
C_{B}^{\Omega} T_{B}^{\Omega}+E_{S}>0 \\
E_{S}>0 \\
C_{B}^{\Omega} T_{B}^{\Omega}>0 \\
\alpha_{q}>0
\end{array}\right\} \Longrightarrow \begin{array}{l}
E_{S}>0 \\
\frac{T_{B}^{\Omega}}{1-q}>0 \\
\frac{1}{1-q}>0
\end{array}\right\} \Longrightarrow q<1
$$

with $T_{B}^{\Omega}>0$. Accordingly, the distribution in Eq. (11) for $q<1$ represents a sharp decay with a cutoff at $E_{\text {tot }}=H_{S}$ in the argument excluding the possibility of fat tails.

In contrast, in terms of the ordinary $q$-exponential distributions, Eq. (9) can be rewritten as

$$
p(\mathbf{x}, \mathbf{p}) \sim \frac{1}{\exp _{q}\left(\epsilon_{q} H_{S}(\mathbf{x}, \mathbf{p})\right)},
$$

with $\epsilon_{q}^{-1}:=(q-1) E_{\mathrm{tot}}$ and

$$
C_{B}^{\Omega}=\frac{1}{q-1} .
$$

With the condition $\epsilon_{q}>0$ now, the following range of validity for the index $q$ is found:

$$
\left.\left.\begin{array}{l}
C_{B}^{\Omega} T_{B}^{\Omega}+E_{S}>0 \\
E_{S}>0 \\
C_{B}^{\Omega} T_{B}^{\Omega}>0 \\
\epsilon_{q}>0
\end{array}\right\} \Longrightarrow \begin{array}{l}
\frac{T_{B}^{\Omega}}{q-1}+E_{S}>0 \\
E_{S}>0 \\
\frac{T_{B}^{\Omega}}{q-1}>0 \\
\frac{1}{q-1}>0
\end{array}\right\} \Longrightarrow q>1
$$

with $T_{B}^{\Omega}>0$. The distribution in Eq. (15) for $q>1$ again represents a sharp decay with a cutoff at $H_{S}=E_{\text {tot }}$ in the argument excluding the possibility of fat tails. These results show that the adoption of ordinary or escort $q$ distributions does not change the form of the probability distribution of the system. The choice between the two aforementioned distributions is only related to the intervals of $q$ values since they are related to one another through the relation $2-q$ (for more details on this issue, see Refs. [21,22]). Note also that the same calculations above can be redone in terms of $C_{B}^{\Phi}$ by using Eq. (8), but it can be observed that the adoption of $C_{B}^{\Phi}$ does not change the shape of the system distribution. The related intervals in Eqs. (12) and (15) change to $q<1 \wedge q>2$ and $q<0 \wedge q>1$, respectively.

\section{B. Case $E_{B}<0$}

This case implies a finite bath with constant negative heat capacity since now $E_{B}=E_{\text {tot }}-H_{S}<0$. To ensure the negativity of the finite bath energy, $E_{\text {tot }}$ must always be negative, so the system distribution does not have a cutoff now. Using again Eqs. (3) and (6), the marginal probability distribution of the system reads

$$
p(\mathbf{x}, \mathbf{p})=c\left(H_{S}(\mathbf{x}, \mathbf{p})-E_{\mathrm{tot}}\right)^{C_{B}^{\Omega}} .
$$

This distribution can be cast into the form of an escort $q$ exponential by the identification (10) so that we have (11) apart from the normalization. Considering consistently the conditions above together with $\alpha_{q}>0$, we obtain the following range of validity for the nonextensivity index $q$ :

$$
\begin{aligned}
& \left.\left.\begin{array}{l}
C_{B}^{\Omega} T_{B}^{\Omega}+E_{S}<0 \\
E_{S}>0 \\
C_{B}^{\Omega} T_{B}^{\Omega}<0 \\
\alpha_{q}>0
\end{array}\right\} \Longrightarrow \begin{array}{l}
\frac{T_{B}^{\Omega}}{1-q}+E_{S}<0 \\
E_{S}>0 \\
\frac{T_{B}^{\Omega}}{1-q}<0 \\
\frac{1}{1-q}<0
\end{array}\right\} \\
& \Longrightarrow 1<q<1+\left(\frac{E_{S}}{T_{B}}\right)^{-1},
\end{aligned}
$$

with $T_{B}^{\Omega}>0$. As can be seen, the term $\frac{E_{S}}{T_{B}}$ has the dimension of a heat capacity. Accordingly, the distribution in Eq. (11) represents an inverse power-law decay with fat tails. This is the result if one agrees to obtain temperature and constant heat capacity of the finite bath in terms of the density of states $\Omega$. In terms of the phase space volume $\Phi$, using Eq. (8), we obtain $C_{B}^{\Phi}=\frac{2-q}{1-q}$, so Eq. (17) yields $1<q<1+\left(\frac{E_{S}}{T_{B}}+1\right)^{-1}$. This result again indicates that the system distribution is an inverse power law with fat tails, although in the thermodynamic limit ( $E_{S} \rightarrow \infty$, so $q$ is confined to the unique value of unity 
implying $C_{B}^{\Omega} \rightarrow-\infty$ ), these $q$ decays are replaced by the usual exponential distribution.

It is worth noting that a particular case of the above general result was also obtained by Lutsko and Boon [23] solely by considering the integration over momenta degrees of freedom so that $E_{S} / T_{B}=D N_{S} / 2$ [compare Eq. (17) above to the one below Eq. (10) in Ref. [23]], where $D$ and $N_{S}$ denote the dimensionality of the phase space and the number of particles in the system, respectively. It is indeed remarkable that Lutsko and Boon obtained this particular result just by checking the integrability conditions of the concomitant Tsallis distributions [23]. However, the results of Lutsko and Boon include neither the finiteness of the bath nor the necessity of its negative heat capacity for the escort $q$ distribution with fat tails to emerge. Due to the generality of the present calculations, one can also consider the influence of including other degrees of freedom on the interval of validity of the nonextensive parameter $q$ : The more the degrees of freedom are associated with the heat capacity of the system, the more the interval of the possible $q$ values is confined. As such, the thermodynamic limit, for any system, corresponds to the unique value of the nonextensivity parameter $q$, i.e., unity, corresponding to the ordinary canonical case.

In contrast, in terms of the ordinary $q$-exponential distributions, Eq. (9) can be rewritten as (13) with (14). With the condition $\epsilon_{q}>0$ now, the following range of validity for the index $q$ is found:

$$
\begin{aligned}
& \left.\left.\begin{array}{l}
C_{B}^{\Omega} T_{B}^{\Omega}+E_{S}<0 \\
E_{S}>0 \\
C_{B}^{\Omega} T_{B}^{\Omega}<0 \\
\epsilon_{q}>0
\end{array}\right\} \Longrightarrow \begin{array}{l}
\frac{T_{B}^{\Omega}}{q-1}+E_{S}<0 \\
E_{S}>0 \\
\frac{T_{B}^{\Omega}}{q-1}<0 \\
\frac{1}{q-1}<0
\end{array}\right\} \\
& \Longrightarrow 1-\left(\frac{E_{S}}{T_{B}}\right)^{-1}<q<1 .
\end{aligned}
$$

The distribution in Eq. (13) represents an inverse power-law decay with fat tails by adopting the density of states $\Omega$. In terms of the phase space volume $\Phi$, using Eq. (8), we have $C_{B}^{\Phi}=$ $\frac{q}{q-1}$, so $1-\left(\frac{E_{S}}{T_{B}}+1\right)^{-1}<q<1$. As expected, when the thermodynamic limit is attained, i.e., $E_{S} \rightarrow \infty$, the value of $q$ assumes only unity, resulting in the usual exponential decay.

Finally, we note that all the results in this section rely on one main ingredient: the dependence of the finite heat capacity of the bath on the nonextensivity parameter $q$ [check Eqs. (10) and (14) for example]. Indeed, this is the sole source for the emergence of the parameter $q$. However, the fact that the bath has constant (positive or negative) heat capacity leaves us with two main possibilities. The first possibility is that the bath is composed of either a finite number of noninteracting particles or particles coupled by a linear harmonic interaction. However, when this is the case, a negative finite heat capacity is physically impossible, so one only has a sharp decay with a cutoff at the total energy for all $q$ intervals certainly different from an inverse power-law decay. The second possibility is that the finite bath might be formed, for example, by a charged particle with constant negative heat capacity (see, for example, the beautifully clear exposition of this case in Ref. [19]). When this is the case, one can indeed have an inverse power-law distribution with fat tails, but this distribution cannot be the Tsallis distribution since its heat capacity, being a negative number, simply does not include any parameter $q$. However, one can choose to write this number (i.e., the constant heat capacity of the finite bath) in terms of a definite $q$ value. This ad hoc identification is not consistent though, since the equipartition theorem is intact even for nonextensive systems $[7,24]$ so the energy and therefore the heat capacity of the finite bath cannot have any explicit $q$ dependence. In other words, it is apparent that the nonextensivity parameter $q$ is merely originating from an illegitimate substitution for the finite heat capacity of the bath as opposed to a possible genuine nonextensivity in the bath.

A related work along this direction is the one by Hasegawa [25], who notes that the nonextensivity index $q$ emerging from the classical finite baths depends mainly on the number of particles in the system and only weakly on the bath degrees of freedom. If the finite baths would be the genuine sources of the Tsallis distributions, the finite heat capacity of the bath should have an explicit $q$ dependence that would be affected by the bath degrees of freedom as usual. That this is not so indicates the validity of the equipartition theorem for all cases, leaving no room for a $q$-dependent constant heat capacity. In short, one can indeed have inverse power-law distributions due to the finiteness of the heat capacity of the bath. However, these inverse power-law distributions are not Tsallis distributions.

\section{CONCLUSION}

There has been a general consensus so far on relating finite baths to the Tsallis distributions. According to this view, the finite baths can have both positive and negative heat capacities depending on the use of ordinary and escort probability distributions [2-4]. It is also held that one can have inverse power-law distributions of Tsallis form with fat tails for all ranges of the nonextensivity parameter $q$.

In order to shed light on all these issues, we have rigorously studied the probability distribution of the system through a microcanonical approach and shown that it stems from the interplay between any arbitrary system and the constant heat capacity of the bath. Only when the bath has finite and constant negative heat capacity, the system attains an inverse powerlaw distribution with fat tails. The finite baths with positive constant heat capacity lead to the system distributions with a well-determined cutoff condition leaving no possibility for the emergence of fat tails.

Whether one adopts the ordinary or escort Tsallis distribution is found to be irrelevant since the choice between the two does not change the nature of the distribution, but only serves for the same distribution to emerge in different intervals of the nonextensivity parameter $q$. Consider, for example, that one has a thermal bath with finite and constant positive heat capacity. Having this information suffices to determine the shape of the system probability distribution, i.e., a sharp decay with a cutoff having no fat tails. However, the adoption of the ordinary Tsallis distribution for this particular case limits the exponent of the Tsallis distribution to the interval $q>1$ [see Eq. (15)], while the same physical case yields $q<1$ for the escort distributions [see Eq. (12)]. Therefore, it is the feature of the finite bath that determines the shape of the system distribution. 
The most important question is finally to decide whether the Tsallis distributions are indeed to emerge from the coupling of the physical system with a finite bath. The answer to this is that they do not since the emergence of the Tsallis distributions in the finite bath scenario, be it ordinary or escort, requires the constant heat capacity of the finite heat bath to be $q$ dependent [see Eqs. (10) and (14) for a complete check]. However, the bath, although finite because of consisting of a finite number of particles but constant nevertheless, leaves us with two options. The first one is that the bath is composed of a finite number of noninteracting particles or particles coupled by linear harmonic interaction. Then it is impossible to realize a negative finite heat capacity for the classical systems. The second option is that the finite bath might be formed, for example, by a charged particle with constant negative heat capacity as in Ref. [19]. This case can indeed lead to an inverse power-law distribution with fat tails, but this distribution cannot be the Tsallis distribution since its heat capacity, being just a number, cannot include any parameter $q$. Despite this, one can still try to write this number i.e., the constant heat capacity of the finite bath, in terms of a definite $q$ value. This ad hoc identification is not consistent though, since the equipartition theorem is intact even for nonextensive systems [7,24], so the energy and therefore the heat capacity of the finite bath cannot have any explicit $q$ dependence. In other words, between these two options, the former cannot yield inverse power-law distributions as necessary for the Tsallis distributions and the latter cannot consistently yield any explicit $q$ dependence in the exponent of the inverse power-law distributions. In short, the inverse power-law distributions are solely due to the finiteness of the heat capacity of the bath and do not admit any $q$ dependence.

One might object to the above conclusion by stating that the interaction energy between the system and the bath has not been taken into account. However, if this is done, the probability distribution is found to be neither exponential nor $q$ exponential [26] [see Eq. (18) therein]. We also note that our results do not exclude the possibility of Tsallis distributions in nonergodic systems such as those composed of classical longrange interacting particles or in some regions of weak chaos [27-30] since the ergodicity of the total system is assumed in the present work.

Considered in the context of recent attempts to $q$ generalized central limit theorems, our results on finite baths seem consistent. There could be no $q$-generalized central limit theorems if one could obtain Tsallis distributions from ordinary classical systems coupled to finite baths. In other words, if the finite baths were the source of genuine Tsallis distributions, one would expect them to emerge from the ordinary law of large numbers as an intermediate distribution despite the lack of correlation. This is apparently not the case [1,27].

\section{ACKNOWLEDGMENTS}

This work was supported by TÜBITAK (Turkey) under Research Project No. 112T083; by the Thales Project MACOMSYS, funded by the ESPA Program of the Ministry of Education of Hellas; and by the European Union's Seventh Framework Programme (Program No. FP7-REGPOT-20122013-1) under Grant No. 316165.
[1] C. Tsallis, Introduction to Nonextensive Statistical Mechanics (Springer, New York, 2009).

[2] A. R. Plastino and A. Plastino, Phys. Lett. A 193, 140 (1994).

[3] M. P. Almeida, Physica A 300, 424 (2001).

[4] J. S. Andrade Jr., M. P. Almeida, A. A. Moreira, and G. A. Farias, Phys. Rev. E 65, 036121 (2002).

[5] F. Q. Potiguar and U. M. S. Costa, Physica A 344, 614 (2004).

[6] M. Campisi, Physica A 385, 501 (2007).

[7] M. Campisi and G. B. Bagci, Phys. Lett. A 362, 11 (2007).

[8] T. S. Biró, Physica A 392, 3132 (2013).

[9] H. Hasegawa, Phys. Rev. E 82, 031138 (2010).

[10] D. Lynden-Bell and R. Wood, Mon. Not. R. Astron. Soc. 138, 495 (1968).

[11] D. Lynden-Bell, Physica A 263, 293 (1999).

[12] W. Thirring, Z. Phys. B 235, 339 (1970).

[13] D. H. E. Gross, Microcanonical Thermodynamics (World Scientific, Singapore, 2001).

[14] S. Hilbert and J. Dunkel, Phys. Rev. E 74, 011120 (2006).

[15] M. Kastner, Phys. Rev. Lett. 104, 240403 (2010).

[16] M. Schmidt, R. Kusche, T. Hippler, J. Donges, W. Kronmüller, B. von Issendorff, and H. Haberland, Phys. Rev. Lett. 86, 1191 (2001).
[17] F. Gobet, B. Farizon, M. Farizon, M. J. Gaillard, J. P. Buchet, M. Carré, P. Scheier, and T. D. Mark, Phys. Rev. Lett. 89, 183403 (2002).

[18] A. I. Khinchin, Mathematical Foundations of Statistical Mechanics (Dover, New York, 1949).

[19] M. Campisi, F. Zhan, and P. Hänggi, Europhys. Lett. 99, 60004 (2012).

[20] J. Dunkel and S. Hilbert, Physica A 370, 390 (2006).

[21] T. Oikonomou and G. B. Bagci, J. Math. Phys. 50, 103301 (2009).

[22] T. Oikonomou and G. B. Bagci, Phys. Lett. A 374, 2225 (2010).

[23] J. F. Lutsko and J. P. Boon, Europhys. Lett. 95, 20006 (2011).

[24] T. S. Biró and P. Ván, Phys. Rev. E 83, 061147 (2011).

[25] H. Hasegawa, Phys. Rev. E 83, 021104 (2011).

[26] J. Jiang et al., Physica A 391, 3140 (2012).

[27] A. Pluchino, A. Rapisarda, and C. Tsallis, Europhys. Lett. 80, 26002 (2007).

[28] M. Leo, R. A. Leo, and P. Tempesta, J. Stat. Mech. (2010) P04021.

[29] M. Leo, R. A. Leo, and P. Tempesta, Ann. Phys. 333, 12 (2013).

[30] L. J. L. Cirto, V. R. V. Assis, and C. Tsallis, Physica A (to be published). 